\title{
PhenoRoots: an inexpensive non-invasive phenotyping system to assess the variability of
} the root system architecture

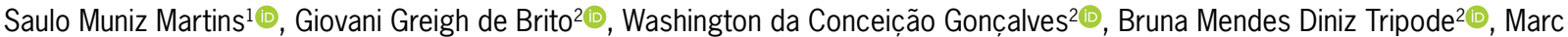

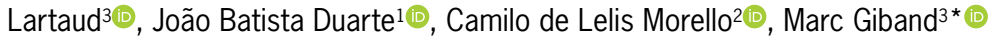

\author{
1Universidade Federal de Goiás/EA - Setor de Genética e \\ Melhoramento de Plantas - Campus Samambaia, Rod. GO \\ 462, km 0 - 74690-900 - Goiânia, GO - Brasil. \\ Embrapa Algodão - Núcleo Cerrado, Rod. GO 462, km \\ 12 - Zona Rural - 75375-000 - Santo Antônio de Goiás, \\ GO - Brasil. \\ ${ }^{3}$ Cirad/UMR AGAP, Av. Agropolis - 34398 - Montpellier \\ Cedex 5 - France. \\ *Corresponding author <marc.giband@cirad.fr>
}

Edited by: Leonardo Oliveira Medici

Received December 28, 2018

Accepted April 02, 2019
ABSTRACT: The root system architecture (RSA) of plants and its functioning play a fundamental role in a number of plant growth mechanisms including water and nutrient uptake. Optimization of the RSA is important for stable and increased plant productivity under adverse conditions. Despite its great importance, studying the RSA is notoriously laborious because of the difficulty of accessing the rooting system of plants. We developed a root phenotyping platform, PhenoRoots, which allows for the non-invasive study of plant RSA. The system was built using inexpensive material and was designed to provide medium throughput. Substrate or soil-filled rhizotrons are used to grow plantlets, whose roots are directly visible through a glass plate. An experiment conducted on a panel of twenty Upland cotton (Gossypium hirsutum L.) varieties demonstrated the usefulness of the platform in assessing RSA traits. A number of traits, destructive and nondestructive, related to the RSA were measured and statistically analyzed. The non-destructive traits based on image analysis of roots were more accurate and showed high correlation with the time-consuming destructive measurements. The platform allowed for capturing the phenotypic and genetic variability found in the panel of cotton varieties, and to define three contrasting RSA patterns. PhenoRoots provides an inexpensive alternative to the medium throughput analysis of RSA traits in plants.

Keywords: rhizotron, root traits, image analysis, medium-throughput, cotton

\section{Introduction}

Because of its role in a number of fundamental processes in plant development, a better understanding of the root system, and its development of complex interactions with the environment, and the genetic control of root system architecture (RSA) holds great promise for plant improvement. Nevertheless, due to the difficulty of securing easy and consistent access, less emphasis has been devoted to the root system than to the aerial part of plants (Herder et al., 2010), and the lack of high performance phenotyping techniques is still a bottleneck to be overcome (Joshi et al., 2017). A number of methods of studying the RSA have been developed, both destructive/invasive and non-destructive methods. The destructive methods basically consist of removing the plant from the soil or from their container in order to weigh the root, both fresh and dry (Shashidhar et al., 2012). These methods are very laborious, cause destruction of the plant and are generally not feasible for large scale experiments. The non-invasive/non-destructive methods of evaluating the RSA are characterized by the use of image processing technologies using "rootboxes" or "rhizotrons" (Neufeld et al., 1989) which facilitate studies throughout the plant's growth and development, and enable evaluation of a large number of genotypes in a much less labor-intensive way.

Advances in the field of root phenotyping have been significant in recent years as several platforms have become available (Bodner et al., 2017; Kuijken et al., 2015). Different approaches have been reported on the characterization of plant RSA, including semi-hydroponics (Chen et al., 2011), hydroponics (Courtois et al., 2013; Mathieu et al., 2015) and soil-based systems (Joshi et al., 2017; Mooney et al., 2012). The "GROWSCREENRhizo" platform (Nagel et al., 2012) is a fully-automated phenotyping system that permits the simultaneous measurement of root and shoot growth for plants grown in soil-filled rhizotrons. However, most of these new technologies remain very expensive and are not suited to most public improvement programs. Bearing this in mind, we developed the "PhenoRoots" platform, a nondestructive, non-invasive, low cost rhizotron-based root phenotyping system, and in this study we present the features and validation of the RSA phenotyping platform. An experiment using 20 Upland cotton (G. hirsutum L.) genotypes was conducted to describe its functionalities, and we discuss its advantages over invasive/ destructive methods of root assessment.

\section{Materials and Methods}

\section{Root phenotyping platform}

The root phenotyping system is constructed using commonly-found and inexpensive material, such as glass and Aluminum Composite Material (ACM) plates, and soldered iron bars. Each rhizotron consists of a 50 $\times 78 \mathrm{~cm}$ light-proof $3.5 \mathrm{~mm}$ thick ACM plate separated from a $50 \times 80 \mathrm{~cm}$ glass plate $(4 \mathrm{~mm}$ thick) by $1.2 \mathrm{~cm}$ thick aluminum spacers. Holes are drilled in the bottom spacer to permit drainage of excess water. The two plates are clamped together using binder clips (Figure 
1A). The sandwich (outer dimension: $1.95 \mathrm{~cm}$, inner dimension: $1.2 \mathrm{~cm}$ ) is filled with substrate and clamped, glass plate side down, on a second ACM plate lined with soft rubber and mounted on a cart. The average $(\mathrm{N}=$ 30) weight of empty, dry substrate-filled, and saturated to field capacity rhizotrons is $6.35 \pm 0.013 \mathrm{~kg}, 9 \pm 0.026$ $\mathrm{kg}$, and $9.6 \pm 0.031 \mathrm{~kg}$, respectively.

The carts ( $2 \mathrm{~m}$ long and $73 \mathrm{~cm}$ high) are built using iron bars that are soldered together and are mounted with wheels to permit easy handling. Each cart holds 10 soft rubber-lined ACM plates on which the rhizotrons are placed, glass plate side down, ensuring that roots grow in light-proof conditions (Figure 1B), while facilitating easy visualization through the glass plate. The ACM supporting plates are positioned at a $45^{\circ}$ angle (Figure 1B), which maximizes the proportion of roots growing against the glass plate to be visible for non-invasive analysis (Figure 2A and B). The platform, composed of 20 carts maintained in a temperature-controlled greenhouse, has the capacity to hold up to 200 rhizotrons.

\section{Analysis of the Root System Architecture and System Validation}

\section{Plant material and growth conditions}

A trial test was conducted in a temperaturecontrolled greenhouse maintained at a day/night tem-

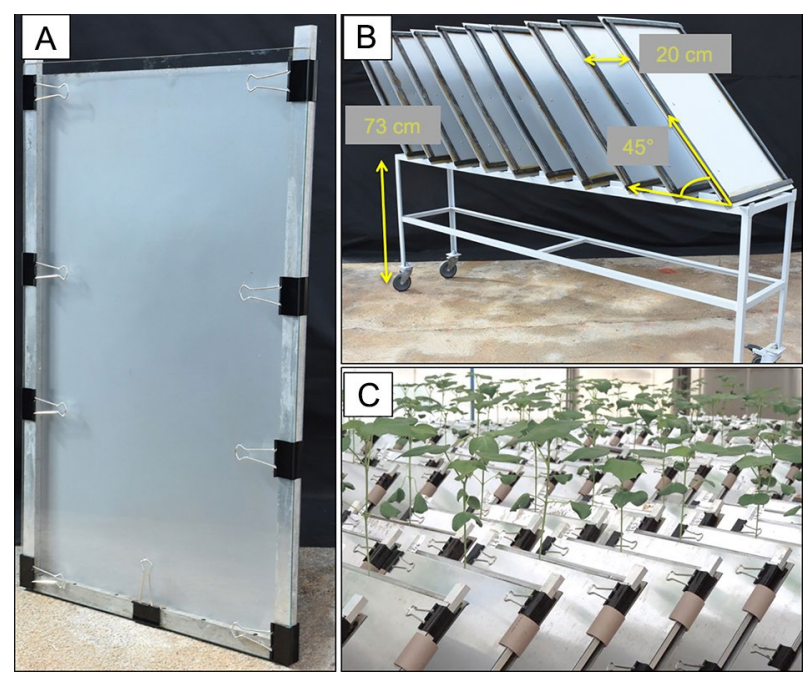

Figure 1 - Overview of the PhenoRoots phenotyping system. A) Rhizotron composed of an Aluminium Composite Material (ACM) light-proof plate (background) separated from a glass plate (foreground) by aluminum spacers. Binder clips are used to clamp the plates together. Note the holes drilled in the bottom spacers to allow excess water to drain. B) Design of the cart used to carry the rhizotrons: rhizotrons are fitted glass-side down against the ACM plates lined with soft rubber, allowing the roots to grow in the dark. C) View of the temperature-controlled greenhouse during a typical PhenoRoots experiment: one cotton plantlet is grown per rhizotron, and maintained until approximately 21 days after sowing. perature of $32 / 22{ }^{\circ} \mathrm{C}$ and an average relative humidity of $70 \%$. Twenty Upland cotton (Gossypium hirsutum L.) varieties (Table 1) were assessed. The experiment was carried out in a randomized complete block design with six replications. Other crop species, including soybean $(G$. max), bean (P. vulgaris), peanut $(A$. hypogaea), corn (Z. mays), rice (O. sativa), sorghum ( $S$. bicolor), and pearl millet (P. glaucum) were also grown in the rhizotrons to demonstrate the versatility of the system.

Each rhizotron was filled with approximately 3 $\mathrm{kg}$ of sieved (4 $\mathrm{mm} \times 4 \mathrm{~mm}$ apertures) potting soil enriched with 4-30-16 NPK fertilizer (375 g per $25 \mathrm{~kg}$ of soil) and ammonium sulfate (100 g per $25 \mathrm{~kg}$ ). During the filling, the glass plate was gently taped with a rubber hammer to ensure even settling of the soil throughout the rhizotron. The soil-filled rhizotrons were then mounted on the carts, and fixed using plastic clamps.

Before sowing, the rhizotrons were saturated by dispensing tap water from the top of the rhizotron, and left to drain until the soil had settled to field capacity. Three seeds were sown per rhizotron the day after the soil had reached field capacity, and the rhizotrons were thinned to one plant per rhizotron by gently removing additional plants with tweezers once the plantlets had emerged.

During the first few days of seed germination, irrigation was maintained so as to keep the soil at field capacity until the plantlets' apexes had emerged from the expanded cotyledonary leaves. This was achieved by manually replenishing the amount of water that had been lost through evapotranspiration. Once the apexes had emerged, irrigation was reduced, and only half of the water lost daily was applied so as to gradually reach $80 \%$ of the soil's field capacity (dry-down). This was usually achieved after a dry-down period of 7-8 days after sowing (DAS), at which point water was supplemented daily to maintain this level of soil moisture in the rhizotrons. To calculate the amount of water lost by evapotranspiration, five additional rhizotrons were randomly allocated in the trial and weighed every day to obtain the average daily loss of water.

Table 1 - List of the Upland cotton varieties used and their respective country of origin.

\begin{tabular}{lccccc}
\hline & Genotype & Country & & Genotype & Country \\
\hline 1 & ACALA MAXXA & USA & 11 & FMT 701 & Brazil \\
2 & ALBAR K603 & Zimbabwe & 12 & FMT 709 & Brazil \\
3 & BRS 269 BURITI & Brazil & 13 & GUAZUNCHO 2 & Argentina \\
4 & BRS 336 & Brazil & 14 & IMACD 8276 & Brazil \\
5 & BRS 372 & Brazil & 15 & IRMA D742 & Cameroon \\
6 & CD 408 & Brazil & 16 & MCU 5 & India \\
7 & CD 410 & Brazil & 17 & N TA 90-8 & Mali \\
8 & FK 290 & Burkina Faso & 18 & REBA P279 & Paraguay \\
9 & FM 966 & Australia & 19 & STAM F & Togo \\
10 & FM 993 & Australia & 20 & STONEVILLE 474 & USA \\
\hline
\end{tabular}




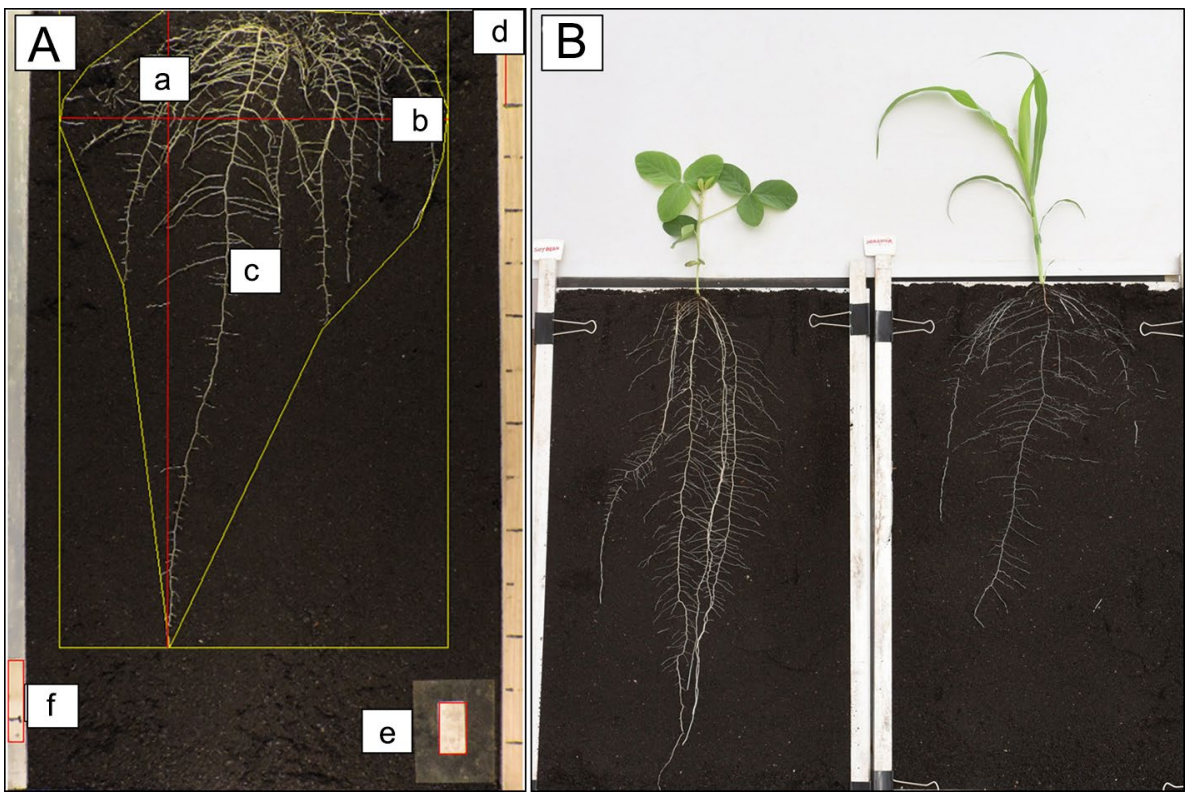

Figure 2 - Representative pictures of the root system as visualized using the PhenoRoots platform. A) Image of the root system of a cotton plant analyzed using the ImageJ toolset: the vertical axis a represents the maximum root depth (mrd); the horizontal axis b represents the maximum root width (mrw); the polygon delineated by the outermost tips of the root system (c) represents the area explored by the roots (ea); ruler (d) with a $5.0 \mathrm{~cm}$ increments; reference (e) of known dimensions $(5 \times 2.5 \mathrm{~cm})$ used to convert pixels into units of length; and label (f) with plot identification. B) Representative pictures of the above-ground and root system of soybean (left) and sorghum (right) plantlets grown on the PhenoRoots platform.

\section{Root picture acquisition, image analysis and RSA traits}

The acquisition of the root images was done at 21 DAS, when the longest roots reached the bottom of the rhizotron. The rhizotrons were removed from the carts and placed, glass plate side up, on a standard photocopy stand equipped with adjustable lighting fitted in a purpose-built dark cabin. The lighting was dimmed and adjusted to a near horizontal position to avoid reflections. Pictures were taken using a digital camera with an 18-megapixel resolution (Nikon Coolpix P520) positioned at a $90^{\circ}$ angle in relation to the plane of the rhizotrons. A ruler with $5.0 \mathrm{~cm}$ increments, a rectangular reference of known size $(2.5 \times 5.0 \mathrm{~cm}$ in our case) and a label indicating plot identification were placed alongside the rhizotron before the pictures were taken (Figure 2A).

Traits related to the root system architecture (RSA) assessed from the 2D RGB images are described in Table 2, and some are illustrated in Figure 2A. Data for the trait total root length (trl), average diameter of roots (adr), number of root tips (tips), projected area (pa), root surface area (rsa) and root volume (rv) were obtained using the WinRHIZO software program (WinRHIZO PRO 2013).

The maximum root depth (mrd), which measures the depth reached by the roots, maximum root width (mrw), and total area explored by the roots (ea) were obtained using a purposely-developed macro toolset running on the ImageJ software program (Abràmoff et al.,
Table 2 - Destructive and non-destructive root system traits assessed in the study, with their respective abbreviation, unit of measure and method used to obtain the data.

\begin{tabular}{lccc}
\hline Trait & Abbreviation & Unit & Data obtained by \\
\hline fresh root weight & frw & $\mathrm{g}$ & Weighing \\
dry root weight & $\mathrm{drw}$ & $\mathrm{g}$ & Weighing \\
\hline total root length & $\mathrm{trl}$ & $\mathrm{cm}$ & WinRHIZO \\
average diameter of roots & $\mathrm{adr}$ & $\mathrm{mm}$ & WinRHIZO \\
number of root tips & $\mathrm{tips}$ & $\mathrm{count}$ & WinRHIZO \\
maximum root depth & $\mathrm{mrd}$ & $\mathrm{cm}$ & ImageJ \\
total explored area & ea & $\mathrm{cm}^{2}$ & ImageJ \\
maximum root width & $\mathrm{mrw}$ & $\mathrm{cm}^{2}$ & ImageJ \\
projected area & pa & $\mathrm{cm}^{2}$ & WinRHIZO \\
root surface area & rsa & $\mathrm{cm}^{2}$ & WinRHIZO \\
root volume & $\mathrm{rv}$ & $\mathrm{cm}^{3}$ & WinRHIZO \\
ratio mrd:mrw & $\mathrm{mrd} \mathrm{mrw}$ & - & Calculation \\
root density (ratio trl:ea) & trl_ea & $\mathrm{cm} \mathrm{cm}^{-2}$ & Calculation \\
\hline
\end{tabular}

2004). A macro file ("Toolset") that contains macro tools available in ImageJ was developed to streamline image analysis and facilitate the semi-automatic measurements of root traits. The toolset includes a calibration tool ("Set Scale" tool from ImageJ) that allows for converting pixels into $\mathrm{cm}$ using the reference of known size (Figure $2 \mathrm{~A}$, letter e). Three tools are used to define the Region of Interest (ROI) for analysing the root system. An area selection tool ("Polygon" tool from ImageJ) enables the total area explored by the roots (ea) to be drawn by click- 
ing on the outermost tips of the rooting system (Figure $2 \mathrm{~A}$ letter $\mathrm{cl}$, and the toolset automatically calculates the maximum root depth (mrd), and the maximum root width (mrw) (Figure 2A, letters a and b respectively). The "Angle" tool from ImageJ allows for measuring the root angle, while the "Segmented Line" tool facilitates measuring the length of any given root. In addition, a counter tool ("Cell Counter" plugin available for ImageJ) enables the counting of objects such as root tips or forks. The "Results" tool activates displaying and saving the image with selections and the ROI Manager in the original image directory. All the measurements are also displayed in the "Analyses" window. The "New" tool allows for resetting the variables and opening the next image. The macro file is available upon request.

In the present study, the root angle tool, the counter tool, and root length tool were not used.

Other traits of interest for the assessment of the RSA are the product of the algebraic relationships between these variables. These include the ratio of mrd to mrw (mrd_mrw) that gives information on the distribution of the roots in the soil profile, and the ratio of trl to ea (trl_ea) which measures root density (Table 2).

At the end of the experiment, destructive measurements including the fresh root weight (frw) and the dry root weight (drw) were obtained. After the photographs had been taken, the rhizotrons were disassembled and the roots collected and washed in a fine-mesh sieve under running tap water. The washed roots were carefully blotted dry to remove excess water and weighed, then dried in an oven to determine the dry weight.

\section{Statistical analysis}

The data were submitted to descriptive and variance analyses, and the coefficient of experimental variation (CVe) was calculated as well as the selective accuracy (SA) using the following formulas: $\mathrm{CVe}=100 \times \sqrt{\mathrm{MSE} / \overline{\mathrm{x}}}$, where MSE is the Mean Squared Error from the ANO$\mathrm{VA}$, and $\mathrm{SA}=\sqrt{1-1 / \mathrm{F}}$, where $\mathrm{F}$ is the value of Snedecor's F test.

Phenotypic means of the varieties were estimated and compared between themselves using the Scott-Knott test. These means were also used to perform Pearson's correlation analysis on relationships between all the variables. Student's t-test was used to test the null hypothesis of the correlations. A heat map and dendogram were generated using the standardized phenotypic mean values of the thirteen RSA traits (Table 2) as measured in the 20 Upland cotton varieties (Table 1) using the "graphics" and "grDevices" packages. All analyses were performed in the $\mathrm{R}$ software environment (R Core Team, 2018).

\section{Results and Discussion}

All the plants grown in the rhizotrons showed normal development and growth of both the shoots and the roots (Figures $1 \mathrm{C}$ and 2A for cotton, and Figure 2B for soybean and sorghum). The positioning of the rhizotron at a $45^{\circ}$ angle induced the roots to grow alongside the glass plate facilitating observation of the rooting system. Additional decreases in the angle (see Figure 1B) did not increase the amount of roots that could be seen (data not shown). The photographs of the root system were taken at the end of the experiment at 21 days after sowing, when the roots of the cotton plants had reached the bottom of the rhizotrons; however, since the system was devised to allow easy handling of the rhizotrons and direct access to the root system, pictures can also be taken repeatedly over the course of the experiment to provide a dynamic view of root growth and development.

Data for eleven image-based root architecture system (RSA) traits and two destructive measurments, namely fresh root weight (frw) and dry root weight (drw), were generated in the trial (Table 2). Analysis of the data showed that the PhenoRoots platform allows for the fast and non-invasive acquisition of robust data with a high degree of experimental accuracy. As shown in Table 3, the platform made it possible to capture the phenotypic variation in RSA traits present in the panel of the 20 Upland cotton varieties. With the exception of the maximum root width (mrw), the traits exhibited moderate to high magnitude variations, with a coefficient of phenotypic variation ranging from $19 \%$ (mrd_mrw) to $60 \%$ (rv) (Table 3).

The degree of experimental precision for the majority of the variables was high, with $\mathrm{CVe}$ ranging from $5 \%$ (mrw) to $33 \%$ (drw) (Table 4). Few traits (pa, rsa, and rv) showed CVe of higher magnitude (39 \%, $39 \%$, and $48 \%$, respectively). It should be noted that these traits were computed using the WinRHIZO software program and are calculated from the "trl" and "adr" variables (Table 2). The degree of experimental precision for the non-destructive traits determined by image analysis was equal to or greater than that of the "frw" and "drw" destructive measurements (Table 4). Thus, the use of image-based methods to measure root system traits, rather than tedious time-and labor-consuming destructive methods, does not lead to lower degrees of experimental precision.

Selective accuracy (SA) has also been proposed as a statistical tool for evaluating the quality of the experiment because it simultaneously considers the magnitude of the residual variation, the number of replicates and the coefficient of genotypic variation (Resende and Duarte, 2007). These authors, consider values above 0.7 as high precision. In our experiment, the magnitude of SA ranged from 0.59 (trl_ea) to 0.87 (mrd), demonstrating that the experiment had a moderate to high degree of precision (Table 4).

Taken together, these results show that the PhenoRoots platform allows for acquiring robust data with a high degree of experimental precision. Data generated through image-based analysis are as precise or more accurate than those generated through destructive measurements, with the added benefit of they being much less labor-intensive and time-consuming. 
Table 3 - Descriptive statistics ${ }^{1}$ of the destructive and the non-destructive image-based traits related to the root system architecture of the Upland cotton varieties.

\begin{tabular}{|c|c|c|c|c|c|c|}
\hline Trait $^{2}$ & Mean & Min & Max & TA & SD & $\mathrm{CV}$ \\
\hline & & & & & & $\%$ \\
\hline frw & 6.05 & 2.30 & 10.27 & 7.97 & 1.77 & 29.19 \\
\hline drw & 0.57 & 0.20 & 1.15 & 0.95 & 0.20 & 35.90 \\
\hline trl & 678.59 & 206.93 & 1404.28 & 1197.35 & 240.14 & 35.39 \\
\hline adr & 1.47 & 0.89 & 2.70 & 1.81 & 0.38 & 25.82 \\
\hline tips & 2736.23 & 1097.00 & 5680.00 & 4583.00 & 793.82 & 29.01 \\
\hline mrd & 59.88 & 22.98 & 71.90 & 48.92 & 11.95 & 19.96 \\
\hline ea & 1347.76 & 495.13 & 2369.31 & 1874.19 & 363.11 & 26.94 \\
\hline mrw & 43.48 & 32.60 & 46.26 & 13.66 & 2.09 & 4.82 \\
\hline pa & 98.53 & 29.21 & 296.22 & 267.01 & 45.47 & 46.15 \\
\hline rsa & 309.53 & 91.77 & 930.60 & 838.83 & 142.85 & 46.15 \\
\hline rv & 11.40 & 2.34 & 32.45 & 30.11 & 6.81 & 59.69 \\
\hline mrd_mrw & 1.38 & 0.52 & 2.08 & 1.56 & 0.27 & 19.35 \\
\hline trl_ea & 0.50 & 0.20 & 0.93 & 0.74 & 0.13 & 25.18 \\
\hline
\end{tabular}

${ }^{1}$ Min = minimum; Max = maximum; TA = total amplitude; SD = standard deviation; CV (\%) = coefficient of phenotypic variation. ${ }^{2 f}$ rw = fresh root weight; drw = dry root weight; trl = total root length; adr = average diameter of roots; tips = number of root tips; $\mathrm{mrd}=$ maximum root depth; ea = total explored area; mrw = maximum root width; $\mathrm{pa}=$ projected area; $r s a=$ root surface area; $r v$ = root volume; mrd_mrw = ratio mrd:mrw; trl_ea = ratio trl:ea (root density).

Table 4 - Summary of the analysis of variance for the destructive and non-destructive image-based traits related to the root system architecture of the Upland cotton varieties.

\begin{tabular}{|c|c|c|c|c|c|c|}
\hline \multirow{2}{*}{ Trait $^{1}$} & \multicolumn{4}{|c|}{ Mean Square } & \multirow{2}{*}{ CVe } & \multirow{2}{*}{ SA } \\
\hline & \multicolumn{2}{|c|}{ Block } & & \multirow[t]{2}{*}{ Genotype } & & \\
\hline & & & & & $\%$ & \\
\hline frw & 4.69 & $0.105^{\text {ns }}$ & 5.37 & $0.005^{* *}$ & 24.41 & 0.77 \\
\hline drw & 0.04 & $0.335^{\text {ns }}$ & 0.06 & $0.072^{\text {ns }}$ & 33.04 & 0.64 \\
\hline trl & 146030.2 & $0.007^{* *}$ & 98222.89 & $0.005^{* *}$ & 30.53 & 0.75 \\
\hline$a d r$ & 0.99 & $0.000^{* *}$ & 0.16 & $0.042^{* *}$ & 20.68 & 0.66 \\
\hline tips & 2049635 & $0.001^{* *}$ & 1091235 & $0.002^{* *}$ & 24.23 & 0.77 \\
\hline mrd & 253.94 & $0.021^{*}$ & 375.25 & 0.000 ** & 15.83 & 0.87 \\
\hline ea & 373434 & $0.004^{* *}$ & 228294.8 & $0.004^{* *}$ & 23.33 & 0.75 \\
\hline mrw & 2.22 & $0.743^{\text {ns }}$ & 6.46 & $0.078^{\text {ns }}$ & 4.65 & 0.61 \\
\hline pa & 10848.4 & $0.000^{* *}$ & 2540.74 & $0.041^{*}$ & 38.52 & 0.66 \\
\hline rsa & 107069 & 0.000 * * & 25075.84 & $0.041^{*}$ & 38.52 & 0.66 \\
\hline rv & 308.65 & $0.000^{* *}$ & 47.64 & $0.084^{\text {ns }}$ & 48.36 & 0.60 \\
\hline mrd_mrw & 0.13 & $0.011^{*}$ & 0.2 & $0.000^{* *}$ & 14.72 & 0.89 \\
\hline trl_ea & 0.02 & $0.194^{\text {ns }}$ & 0.02 & $0.094^{\mathrm{ns}}$ & 23.77 & 0.59 \\
\hline
\end{tabular}

${ }_{1}^{1}$ frw = fresh root weight; drw = dry root weight; trl = total root length; $\mathrm{adr}=$ average diameter of roots; tips = number of root tips; $\mathrm{mrd}=$ maximum root depth; ea = total explored area; mrw = maximum root width; $\mathrm{pa}=$ projected area; $r s a=$ root surface area; $r v=$ root volume; mrd_mrw = ratio mrd:mrw; trl_ea $=$ ratio trl:ea (root density). ${ }^{\text {ns }},{ }^{*},{ }^{* *}=$ not significant, significant at $p<$ 0.05 and significant at $p<0.01$ respectively. $\mathrm{CVe}=$ coefficient of experimental variation. $\mathrm{SA}=$ Selective accuracy.

The data generated by this phenotyping platform allowed for capturing the genetic variability of cotton RSA traits present in the panel of varieties that were assessed. For nine of the traits (frw, trl, adr, tips, mrd, ea, pa, rsa and mrd_mrw), a significant difference $(p<0.05)$ was observed between the genotypes (Table 4).

To exemplify the variability in root system traits found in this panel of varieties, the results of the phenotypic values (mean values over the six repetitions) are presented in Table 5. Once more, except for a few traits (drw, adr, mrw, rv, and trl_ea), differences were found between the varieties of cotton. The following varieties Reba P279, Stam F and Guazuncho 2 on the one hand, and IMACD 8276, FM 966, CD 410, FMT 701 and BRS 336 on the other, had markedly contrasting root systems. The former three varieties presented higher values than the subsequent five in eight of the thirteen traits assessed (Table 5). Thus, these three varieties, mainly Reba P279, displayed a more robust root system, through a more expanded area of contact with the soil, a more extensive area explored by the root system and a greater depth reached.

The heat map generated after a multivariate analysis using the standardized mean values of the thirteen traits defined three clusters of varieties with differing patterns of root systems (Figure 3). A first cluster, comprising the varieties Reba P279, Stam F and Guazuncho 2, displayed a rooting system with a dominant tap root, and a deeper root system exploring a greater area. A second cluster, composed of varieties CD 410, BRS 336, FMT 701, IMACD 8276 and FM 966, displayed more superficial and ramified roots. The remaining varieties consisted of a third cluster with an intermediate RSA.

Using image-based data generated on the root phenotyping platform described in this study, strong positive and significant correlation between the variables obtained by non-invasive root image analysis and variables obtained only by destructive and time-consuming methods, such as root fresh and dry weight, were observed (Table 6). For example, the destructive trait "fresh root weight" (frw) showed a series of significant high positive correlation with the image-based variables "total explored area" (ea) and "maximum root width" (mrw) values measured using the ImageJ software program as well as with the variable "total root length" (trl) as measured by the WinRHIZO software program $(0.69,0.73$, 
Table 5 - Adjusted means of the traits ${ }^{1}$ related to the root system architecture of the Upland cotton varieties.

\begin{tabular}{|c|c|c|c|c|c|c|c|c|c|c|c|c|c|}
\hline Variety & frw & drw & trl & adr & tips & $\mathrm{mrd}$ & ea & mrw & $\mathrm{pa}$ & rsa & rv & mrd_mrw & trl_ea \\
\hline REBA P279 & $8.21 \mathrm{a}$ & $0.75 a$ & 969.85 a & $1.42 \mathrm{a}$ & $3668 \mathrm{a}$ & $67.50 \mathrm{a}$ & $1717 \mathrm{a}$ & $44.36 \mathrm{a}$ & $139.33 \mathrm{a}$ & $437.71 \mathrm{a}$ & $16.32 \mathrm{a}$ & $1.52 \mathrm{a}$ & $0.52 \mathrm{a}$ \\
\hline STAM F & $7.81 \mathrm{a}$ & $0.66 \mathrm{a}$ & 890.88 a & $1.58 \mathrm{a}$ & 3367 a & 66.84 a & 1679 a & $44.12 \mathrm{a}$ & $141.21 \mathrm{a}$ & 443.62 a & $17.77 \mathrm{a}$ & $1.51 \mathrm{a}$ & $0.54 \mathrm{a}$ \\
\hline CD 408 & $7.24 \mathrm{a}$ & $0.70 \mathrm{a}$ & $728.79 b$ & $1.51 \mathrm{a}$ & $2763 b$ & $56.20 \mathrm{~b}$ & $1273 b$ & 43.78 a & $111.04 \mathrm{a}$ & 348.84 a & $13.86 \mathrm{a}$ & $1.29 \mathrm{~b}$ & $0.54 \mathrm{a}$ \\
\hline FM 993 & $7.21 \mathrm{a}$ & $0.69 a$ & $740.69 \mathrm{~b}$ & $1.24 \mathrm{a}$ & $3029 a$ & $55.80 \mathrm{~b}$ & $1359 \mathrm{~b}$ & $43.23 \mathrm{a}$ & $91.61 \mathrm{~b}$ & 287.81 b & $8.94 \mathrm{a}$ & $1.29 \mathrm{~b}$ & $0.53 \mathrm{a}$ \\
\hline GUAZUNCHO 2 & $6.93 a$ & $0.60 a$ & 941.34 a & $1.64 \mathrm{a}$ & 3660 a & $65.47 \mathrm{a}$ & 1561 a & 43.93 a & 135.93 a & 427.03 a & $10.27 \mathrm{a}$ & $1.49 \mathrm{a}$ & $0.62 \mathrm{a}$ \\
\hline FMT 701 & $6.61 \mathrm{a}$ & $0.65 a$ & $569.32 \mathrm{~b}$ & $1.24 \mathrm{a}$ & $2278 b$ & $41.58 \mathrm{~b}$ & $1157 \mathrm{~b}$ & $43.65 \mathrm{a}$ & 71.84 b & $225.70 \mathrm{~b}$ & $7.26 \mathrm{a}$ & $0.96 \mathrm{c}$ & $0.50 \mathrm{a}$ \\
\hline BRS 269 BURITI & $6.49 a$ & $0.76 \mathrm{a}$ & $604.84 \mathrm{~b}$ & $1.30 \mathrm{a}$ & $2574 b$ & $51.69 \mathrm{~b}$ & $1156 b$ & 43.68 a & $79.51 \mathrm{~b}$ & $249.80 \mathrm{~b}$ & $8.55 \mathrm{a}$ & $1.18 \mathrm{~b}$ & $0.52 \mathrm{a}$ \\
\hline N’TA 90-8 & $6.18 \mathrm{a}$ & $0.55 a$ & $611.44 b$ & $1.42 \mathrm{a}$ & 2577 b & $67.04 \mathrm{a}$ & 1472 a & $43.85 \mathrm{a}$ & $86.89 \mathrm{~b}$ & $272.98 b$ & $9.80 \mathrm{a}$ & $1.53 \mathrm{a}$ & $0.42 \mathrm{a}$ \\
\hline FK 290 & $6.11 \mathrm{a}$ & $0.61 \mathrm{a}$ & 658.74 b & $1.41 \mathrm{a}$ & $2791 b$ & 63.93 a & 1656 a & $44.55 \mathrm{a}$ & $95.11 b$ & $298.79 b$ & $11.05 \mathrm{a}$ & $1.44 \mathrm{a}$ & $0.40 a$ \\
\hline FMT 709 & $6.11 \mathrm{a}$ & $0.54 \mathrm{a}$ & 702.36 b & $1.35 \mathrm{a}$ & $2859 \mathrm{~b}$ & $58.46 a$ & $1253 b$ & $43.21 \mathrm{a}$ & $95.36 \mathrm{~b}$ & $299.58 b$ & $10.94 \mathrm{a}$ & $1.35 \mathrm{~b}$ & $0.57 \mathrm{a}$ \\
\hline ACALA MAXXA & $5.97 \mathrm{a}$ & $0.57 a$ & $661.53 b$ & $1.60 \mathrm{a}$ & $2733 b$ & $63.07 \mathrm{a}$ & $1387 b$ & 44.59 a & $102.17 b$ & $320.98 b$ & $12.80 \mathrm{a}$ & $1.41 \mathrm{a}$ & $0.47 a$ \\
\hline ALBAR K603 & $5.97 \mathrm{a}$ & $0.60 \mathrm{a}$ & $637.90 \mathrm{~b}$ & $1.41 \mathrm{a}$ & $2576 b$ & $58.80 \mathrm{a}$ & $1309 \mathrm{~b}$ & 44.47 a & $93.99 \mathrm{~b}$ & $295.28 b$ & $11.15 \mathrm{a}$ & $1.32 \mathrm{~b}$ & $0.48 \mathrm{a}$ \\
\hline IRMA D742 & $5.95 a$ & $0.46 a$ & $590.45 b$ & $1.91 \mathrm{a}$ & $2558 b$ & 66.49 a & 1446 a & $44.41 \mathrm{a}$ & $103.93 b$ & $326.50 \mathrm{~b}$ & $15.30 \mathrm{a}$ & $1.50 \mathrm{a}$ & $0.42 \mathrm{a}$ \\
\hline BRS 372 & $5.72 \mathrm{a}$ & $0.60 \mathrm{a}$ & $811.11 \mathrm{a}$ & $1.49 \mathrm{a}$ & $3075 a$ & $69.06 \mathrm{a}$ & $1347 b$ & $43.78 \mathrm{a}$ & $121.69 \mathrm{a}$ & $382.31 \mathrm{a}$ & $10.27 \mathrm{a}$ & $1.66 \mathrm{a}$ & $0.57 \mathrm{a}$ \\
\hline BRS 336 & $5.31 b$ & $0.53 a$ & $472.04 b$ & $1.46 \mathrm{a}$ & $2155 b$ & 65.88 a & $1253 b$ & $43.57 \mathrm{a}$ & $64.60 \mathrm{~b}$ & $202.96 b$ & $7.29 a$ & $1.51 \mathrm{a}$ & $0.37 a$ \\
\hline CD 410 & $5.16 b$ & $0.43 a$ & $580.04 \mathrm{~b}$ & $1.69 \mathrm{a}$ & 2269 b & $67.06 \mathrm{a}$ & $1267 b$ & $42.70 \mathrm{a}$ & $96.44 \mathrm{~b}$ & $302.98 b$ & $13.55 \mathrm{a}$ & $1.57 \mathrm{a}$ & $0.46 a$ \\
\hline MCU 5 & $5.12 b$ & $0.50 \mathrm{a}$ & $687.67 b$ & $1.37 \mathrm{a}$ & 2764 b & $63.40 \mathrm{a}$ & $1385 b$ & $42.49 a$ & $93.15 b$ & $292.63 b$ & $10.36 \mathrm{a}$ & $1.49 \mathrm{a}$ & $0.50 \mathrm{a}$ \\
\hline STONEVILLE 474 & $4.71 b$ & $0.39 a$ & $641.92 b$ & $1.48 \mathrm{a}$ & $2616 b$ & $54.28 b$ & $1172 b$ & 43.27 a & $94.92 \mathrm{~b}$ & $298.20 b$ & $11.91 \mathrm{a}$ & $1.25 b$ & $0.53 \mathrm{a}$ \\
\hline FM 966 & $3.72 b$ & $0.32 \mathrm{a}$ & $503.69 \mathrm{~b}$ & $1.46 \mathrm{a}$ & $1980 \mathrm{~b}$ & $50.39 b$ & $1038 b$ & $40.73 \mathrm{a}$ & $78.99 b$ & $248.16 b$ & $10.76 \mathrm{a}$ & $1.22 \mathrm{~b}$ & $0.49 a$ \\
\hline IMACD 8276 & $3.49 \mathrm{~b}$ & $0.37 \mathrm{a}$ & $539.80 \mathrm{~b}$ & $1.25 \mathrm{a}$ & $2343 b$ & $46.19 \mathrm{~b}$ & $1060 \mathrm{~b}$ & $41.20 \mathrm{a}$ & $68.89 \mathrm{~b}$ & $216.43 b$ & $7.17 \mathrm{a}$ & $1.00 \mathrm{c}$ & $0.47 \mathrm{a}$ \\
\hline
\end{tabular}

Table 6 - Phenotypic linear correlation for the traits ${ }^{1}$ related to the root system architecture measured in the Upland cotton varieties.

\begin{tabular}{|c|c|c|c|c|c|c|c|c|c|c|c|c|}
\hline & frw & drw & trl & $\mathrm{adr}$ & tips & mrd & ea & mrw & pa & rsa & rv & mrd_mrw \\
\hline drw & $0.89^{* *}$ & & & & & & & & & & & \\
\hline $\operatorname{trl}$ & $0.72^{* *}$ & $0.56^{*}$ & & & & & & & & & & \\
\hline $\mathrm{adr}$ & $0.05^{\text {ns }}$ & $-0.24^{\mathrm{ns}}$ & $0.12^{\mathrm{ns}}$ & & & & & & & & & \\
\hline tips & $0.73^{* *}$ & $0.58^{* *}$ & $0.98^{* *}$ & $0.11^{\mathrm{ns}}$ & & & & & & & & \\
\hline mrd & $0.32^{\mathrm{ns}}$ & $0.13^{\text {ns }}$ & $0.45^{*}$ & $0.61^{* *}$ & $0.48^{*}$ & & & & & & & \\
\hline ea & $0.69^{* *}$ & $0.48^{*}$ & $0.72^{* *}$ & $0.33^{\text {ns }}$ & $0.77^{* *}$ & $0.73^{* *}$ & & & & & & \\
\hline mrw & $0.73^{* *}$ & $0.67^{* *}$ & $0.43^{\mathrm{ns}}$ & $0.30^{\text {ns }}$ & $0.50^{*}$ & $0.50^{*}$ & $0.67^{* *}$ & & & & & \\
\hline $\mathrm{pa}$ & $0.64^{* *}$ & $0.40^{\text {ns }}$ & $0.92^{* *}$ & $0.46^{*}$ & $0.88^{* *}$ & 0.60 ** & $0.75^{* *}$ & $0.47^{*}$ & & & & \\
\hline rsa & $0.64^{* *}$ & $0.40^{\text {ns }}$ & $0.92^{* *}$ & $0.46^{*}$ & $0.88^{* *}$ & $0.60^{* *}$ & $0.75^{\star *}$ & $0.47^{\star}$ & $1.00^{* *}$ & & & \\
\hline rv & $0.45^{*}$ & $0.14^{\mathrm{ns}}$ & $0.52^{*}$ & $0.64^{* *}$ & $0.46^{\star}$ & $0.52^{*}$ & $0.59^{* *}$ & $0.38^{\text {ns }}$ & $0.76^{* *}$ & $0.76^{* *}$ & & \\
\hline mrd_mrw & $0.27^{\mathrm{ns}}$ & $0.09^{\text {ns }}$ & $0.42^{\mathrm{ns}}$ & $0.59^{* *}$ & $0.43^{\text {ns }}$ & $0.98^{* *}$ & $0.66^{* *}$ & $0.41^{\text {ns }}$ & $0.57^{* *}$ & $0.57^{* *}$ & $0.49^{*}$ & \\
\hline trl_ea & $0.32^{\mathrm{ns}}$ & $0.28^{\text {ns }}$ & $0.69^{* *}$ & $-0.10^{\text {ns }}$ & $0.62^{* *}$ & $-0.11^{\text {ns }}$ & $0.03^{\text {ns }}$ & $-0.04^{\text {ns }}$ & $0.57^{* *}$ & $0.57^{* *}$ & $0.14^{\mathrm{ns}}$ & $-0.06^{\mathrm{ns}}$ \\
\hline
\end{tabular}

${ }_{1}$ frw = fresh root weight; drw = dry root weight; trl = total root length; adr = average diameter of roots; tips = number of root tips; mrd = maximum root depth; ea = total explored area; $\mathrm{mrw}=$ maximum root width; $\mathrm{pa}=$ projected area; $r \mathrm{sa}=$ root surface area; $r v=$ root volume; mrd_mrw = ratio mrd:mrw; trl_ea = ratio trl:ea (root density). ns, ${ }^{*},{ }^{* *}=$ not significant, significant at $p<0.05$ and significant at $p<0.01$ respectively.

and 0.72 , respectively) (Table 6). This underlines the potential of the platform to make a phenotypic assessment of the root system architecture of plants.

While developing the PhenoRoots system, we struck a compromise between the size of the rhizotron, and thus its capacity to allow plant growth over an extended period of time, and the ease of handling, and thereby the throughput of the platform. The $80 \mathrm{~cm}$-high glass plates enabled cotton plants to grow for 21 days after sowing until the longest tap roots reached the bottom of the rhizotron, a stage at which cotton plants were still at a vegetative stage. Souza et al. (1983) have never- theless shown that the selection of cotton plants at the seedling stage results in longer roots in adult plants, and the period of growth on the platform is therefore adequate for this purpose. If necessary, the rhizotrons may be resized and the platform adapted to fit larger plates to allow for the monitoring of root development over longer periods of time. However, increasing the dimensions of the rhizotrons results in increased weight and reduced ease of handling, and may affect the throughput of the system.

We successfully tested the ability of the platform to support the growth of other crop species, including 


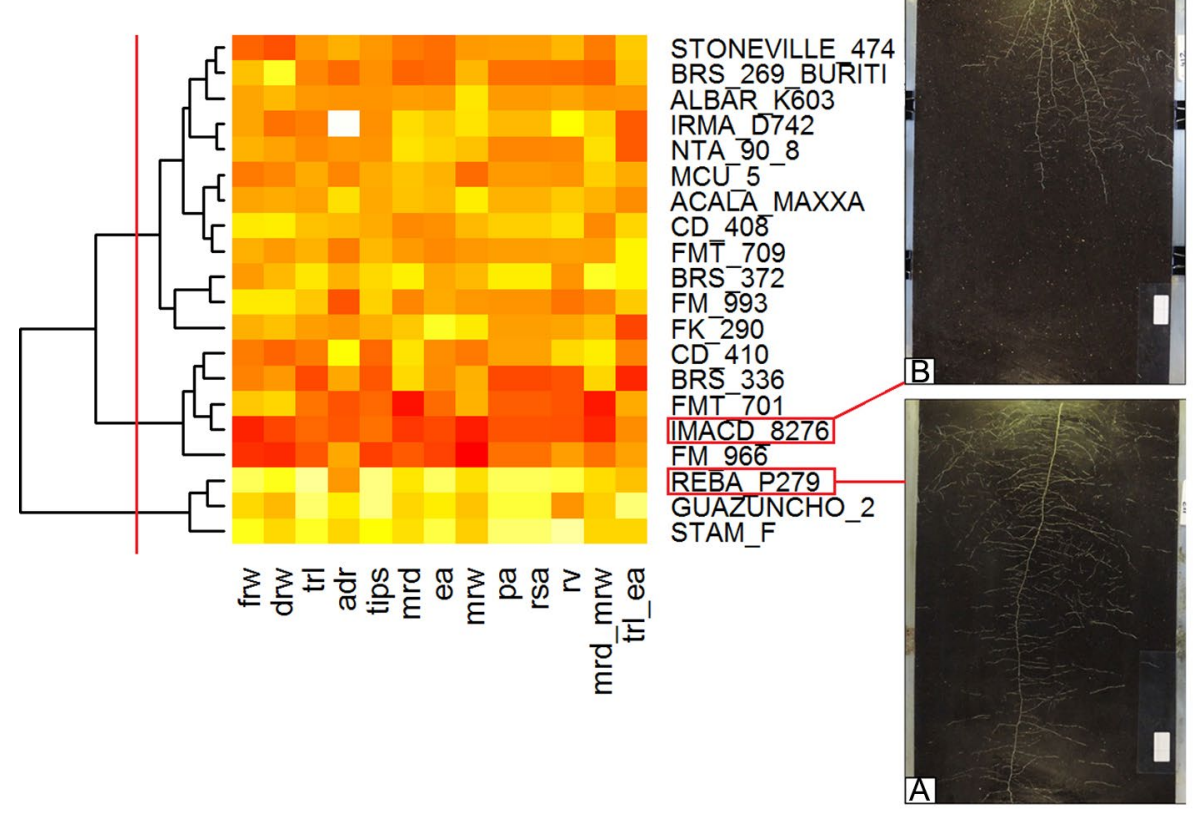

Figure 3 - Heat map and dendogram showing the cluster analysis generated using the standardized mean values of the thirteen root system architecture traits ${ }^{1}$ measured in 20 Upland cotton varieties. Lighter colors indicate higher trait values while darker colors indicate lower values. The panels on the right show typical pictures of the root system of a variety (Reba P279) displaying a deeper system that explores a greater soil area (panel A), while panel B shows a variety (IMACD 8276) with a more superficial root system. ${ }^{1}$ frw $=$ fresh root weight; drw = dry root weight; trl = total root length; adr = average diameter of roots; tips = number of root tips; $\mathrm{mrd}=$ maximum root depth; ea = total explored area; $\mathrm{mrw}=$ maximum root width; $\mathrm{pa}=$ projected area; $r s a=$ root surface area; $r \mathrm{v}=$ root volume; mrd_mrw = ratio mrd:mrw; trl_ea = ratio trl:ea (root density).

soybean (G. max), bean (P. vulgaris), peanut (A. hypogaea), corn (Z. mays), rice (O. sativa), sorghum (S. bicolor), and pearl millet $(P$. glaucum). Figure $2 \mathrm{~B}$ shows the above-ground and the root development of soybean (left) and sorghum (right) plantlets. As for cotton, plantlet growth and development was satisfactory. In addition to the root system architecture traits that were assessed in this study on cotton, the ImageJ toolset that was developed allows for the measurement of other RSA features. In particular, in grasses such as rice (Kato et al., 2006), wheat (Manschadi et al., 2008), barley (Hargreaves et al., 2009) and maize (Singh et al., 2010), the root growth angle, which can also easily be measured using this system (not shown), has been shown to be important in a number of aspects related to plant growth and tolerance to abiotic stresses.

The "PhenoRoots" platform, a low cost root phenotyping system allows for the non-destructive analysis of RSA traits. Using Upland cotton as an example, we show that the platform facilitates capturing the phenotypic and genetic variability for the RSA traits under study present in our panel of varieties. More importantly, we showed that non-destructive image-based analyses display high correlation with destructive and timeconsuming measurements.

In our particular setup, we are able to evaluate up to 200 plants in a single experiment, with an estimated hands-on time of 3-4 min per plant to document the root system. Image analysis could then be carried out over time using freely- or commercially-available image/root analysis software. This level of throughput is compatible with what is necessary in the screening and pre-breeding stages of a plant improvement program. In addition to the application described in this study, we anticipate that the PhenoRoots platform will also be useful to a study of the dynamics of root system development over time, and the effect of abiotic stresses (salt, nutrient, or water stress) on root development. As such, by facilitating the integration of phenotypic, genotypic and genomic approaches, low-cost versatile root phenotyping systems such as the PhenoRoots platform will help better apprehend the complex interactions that shape plant root systems and their adaptive responses to environmental changes.

\section{Acknowledgments}

SMM holds a PhD scholarship from CAPES /Coordenação de Aperfeiçoamento de Pessoal de Nível Superior, Brazil). This study was conducted within the framework of an Agropolis Fondation/Empresa Brasileira de Pesquisa Agropecuária (Embrapa)/CAPES project granted to MG, CLM and JBD and received support from the Agropolis Fondation under reference ID 1402-006 
through the "Investissements d'avenir" program (Labex Agro:ANR-10-LABX-0001-01). We thank the Instituto Mato Grossense do Algodão (IMAmt, Brazil) for partial funding of this research, and Sebastião Araújo for photographic work.

\section{Authors' Contributions}

Conceptualization: Brito, G.G.; Tripode, B.M.D.; Giband, M. Data acquisition: Martins, S.M.; Gonçalves, W.C.; Tripode, B.M.D.; Giband, M. Data analysis: Martins, S.M.; Duarte, J.B.; Giband, M. Design of methodology: Martins, S.M.; Duarte, J.B.; Morello, C.L.; Giband, M. Image analysis toolset development: Lartaud, M. Writing and editing: Martins, S.M.; Brito, G.G.; Duarte, J.B.; Morello, C.L.; Giband, M.

\section{References}

Abràmoff, M.D.; Magalhães, P.J.; Ram, S.J. 2004. Image processing with ImageJ. Biophotonics International 11: 36-42.

Bodner, G.; Alsalem, M.; Nakhforoosh, A.; Arnold, T.; Leitner, D. 2017. RGB and spectral root imaging for plant phenotyping and physiological research: experimental setup and imaging protocols. Journal of Visualized Experiments 126: 1-21.

Chen, Y.L.; Dunbabin, V.M.; Diggle, A.J.; Siddique, K.H.M.; Rengel, Z. 2011. Development of a novel semi-hydroponic phenotyping system for studying root architecture. Functional Plant Biology 38: 355-363.

Courtois, B.; Audebert, A.; Dardou, A.; Roques, S.; GhneimHerrera, T.; Droc, G.; Frouin, J.; Rouan, L.; Gozé, E.; Kilian, A.; Ahmadi, N.; Dingkuhn, M. 2013. Genome wide association mapping of root traits in a japonica rice panel. PLoS One 8: e78037.

Hargreaves, C.E.; Gregory, P.J.; Bengough, A.G. 2009. Measuring root traits in barley (Hordeum vulgare ssp. vulgare and ssp. spontaneum) seedlings using gel chambers, soil sacs and X-ray microtomography. Plant Soil 316: 285-297.

Herder, G.D.; van Isterdael, G.; Beeckman, T.; De Smet, I. 2010. The roots of a new green revolution. Trends in Plant Science 15: 600-607.

Joshi, D.C.; Singh, V.; Hunt, C.; Mace, E.; van Oosterom, E.; Sulman, R.; Jordan, D.; Hammer, G. 2017. Development of a phenotyping platform for high throughput screening of nodal root angle in sorghum. Plant Methods 13, 56: 1-12.
Kato, Y.; Abe, J.; Kamoshita, A.; Yamagishi, J. 2006. Genotypic variation in root growth angle in rice and its association with deep root development in upland fields with different water regimes. Plant Soil 287: 117-129.

Kuijken, R.C.P.; van Eeuwijk, A.; Marcelis, L.F.M.; Bouwmeester, H.J. 2015. Root phenotyping: from component trait in the lab to breeding. Journal of Experimental Botany 66: 5389-5401.

Manschadi, A.M.; Hammer, G.L.; Christopher, J.T.; deVoil, P. 2008. Genotypic variation in seedling root architectural traits and implications for drought adaptation in wheat (Triticum aestivum L.). Plant Soil 303: 115-129.

Mathieu, L.; Lobet, G.; Tocquin, P.; Périlleux, C. 2015. "Rhizoponics": A novel hydroponic rhizotron for root system analyses on mature Arabidopsis thaliana plants. Plant Methods 11: $1-7$.

Mooney, S.J.; Pridmore, T.P.; Helliwell, J.; Bennett, M.J. 2012. Developing X-ray computed tomography to non-invasively image 3-D root systems architecture in soil. Plant Soil 352: 1-22.

Nagel, K.A.; Putz, A.; Gilmer, F.; Heinz, K.; Fischbach, A.; Pfeifer, J.; Faget, M.; Blossfeld, S.; Ernst, M.; Dimaki, C.; Kastenholz, B.; Kleinert, A.K.; Galinski, A.; Scharr, H.; Fiorani, F.; Schurr, U. 2012. GROWSCREEN-Rhizo is a novel phenotyping robot enabling simultaneous measurements of root and shoot growth for plants grown in soil-filled rhizotrons. Functional Plant Biology 39: 891-904.

Neufeld, H.S.; Durall, D.M.; Rich, P.M.; Tingey, D.T. 1989. A rootbox for quantitative observations on intact entire root systems. Plant and Soil 117: 295-298.

Resende, M.D.V.; Duarte, J.B. 2007. Precision and quality control in variety trials. Pesquisa Agropecuária Tropical 37: 182-194 (in Portuguese, with abstract in English).

Shashidhar, H.E.; Henry, A.; Hardy, B. 2012. Methodologies for Root Drought Studies in Rice. International Rice Research Institute, Los Baños, Philippines.

Singh, V.; van Oosterom, E.J.; Jordan, D.R.; Messina, C.D.; Cooper, M.; Hammer, G.L. 2010. Morphological and architectural development of root systems in sorghum and maize. Plant Soil 333: 287-299.

Souza, J.G.; Silva, J.B.V.; Barreiro Neto, M.; Giles, J.A. 1983. Speed of growth as a criterion of drought resistance in cotton. Pesquisa Agropecuária Brasileira 18: 169-172 (in Portuguese, with abstract in English). 